\title{
PERFIL DE RIESGOS DE CONTAMINACIÓN MICROBIOLÓGICA Y QUÍMICA EN LA CADENA DE PRODUCCIÓN DE NUEVE PRODUCTOS HORTÍCOLAS PARA CONSUMO FRESCO, DE UN GRUPO DE EMPRESAS AGRÍCOLAS DEL VALLE CENTRAL DE COSTA RICA ${ }^{1}$
}

\author{
Guillermo Vargas-Hernández $z^{2 *}$, Alfredo Durán-Quirós*, María Isabel González-Lutz**, \\ Dennis Mora-Acedo*
}

Palabras clave: Inocuidad; procesos; riesgo; BPA; mercado. Keywords: Safety; processes; risk; GAP; market.

Recibido: 05/03/15

\section{RESUMEN}

Se determinó el riesgo de contaminación microbiológica o química en cada uno de los procesos de los sistemas de producción de hortalizas de consumo fresco, en una población de 164 empresas agrícolas del Valle Central de Costa Rica. La cuantificación del riesgo se calculó por medio de una encuesta de 46 ítems realizada a los gerentes de las empresas incluidas en el estudio. Las respuestas de los 46 ítems fueron agrupadas según el proceso de producción; se utilizó la fórmula para el cálculo del riesgo de contaminación validada por los autores en otra investigación y se obtuvo el valor de riesgo por proceso. Se calcularon los resultados del riesgo de contaminación microbiológica o química para el proceso de preparación de terreno y siembra, manejo cultural del cultivo, factor humano que realiza el manejo cultural del cultivo, control fitosanitario, cosecha, empaque y para el factor humano ligado al empaque. Los resultados del análisis de riesgo indican que el factor humano que participa en el empaque obtiene la calificación de riesgo más alta, seguido por el proceso de manejo cultural del cultivo; luego aparece el proceso de cosecha.

\footnotetext{
1 Parte del Proyecto de investigación "Diagnóstico in situ de la situación de la inocuidad de la producción y manejo de los principales cultivos hortícolas de consumo fresco para exportación y consumo en el país". Universidad de Costa Rica, 2013.

Aceptado: 28/05/15

\begin{abstract}
Risk profile of microbiological and chemical contamination in the production chain of nine horticultural products for fresh consumption of a group of agricultural companies in the Costa Rican Central Valley. The risk of microbiological or chemical contamination was determined in each of the processes of the production system of produce for fresh consumption, in a population of 164 agricultural companies in the central valley of Costa Rica. The risk quantification was calculated using the responses to a 46-item survey conducted with the managers of the companies included in the study. The responses to the 46 items were grouped according to the production process; the risk contamination calculation formula validated by the authors in another study was used to obtain the risk value per process. The microbiological or chemical contamination risk results were calculated for the land preparation and planting process, cultural management of the crop, human factor performing the cultural management of the crop, phytosanitary control, harvesting, packaging, and the human factor

\footnotetext{
* Estación Experimental Fabio Baudrit, Facultad de Ciencias Agroalimenarias, Universidad de Costa Rica.

** Escuela de Estadística, Facultad de Ciencias Económicas. Universidad de Costa Rica.
} 
En todos los procesos donde se cuantificó el riesgo, los resultados presentan valores cercanos o superiores a 50 en una escala de riesgo de 0 a 100. Estos resultados deben alertar a los productores y consumidores de hortalizas para consumo fresco de la población analizada. Se observó además el efecto del comprador sobre los valores de riesgo en el empaque; cuando el productor es proveedor de una cadena de supermercados, los valores de riesgo disminuyen al compararlos con productores que no son proveedores de esas cadenas.

\section{INTRODUCCIÓN}

Actualmente, los sistemas de producción agrícola están influenciados por diversas fuerzas de mercado producto del impacto de la globalización, esto ha obligado a las empresas a ser más competitivas con el objetivo de permanecer en el mercado (Porter 2009).

Aspectos como rentabilidad, sostenibilidad ambiental, eficiencia en la producción, bienestar social, innovación e inocuidad de los productos agrícolas que produce, son sólo algunos factores que se suman a los requisitos que debe dominar una empresa agrícola competitiva en la actualidad, y son algunas de las fuerzas de mercado que se indicaron anteriormente (Brown 2009, Amekawa 2009, Herzfeld et ál. 2011).

La pérdida de la inocuidad es un riesgo alto, no sólo para los clientes finales que consumen los alimentos; provoca además una serie de consecuencias negativas, tanto para la empresas agrícolas que lo producen y lo llevan al mercado, como para las relaciones comerciales internacionales del país de origen, tal como lo indican FAO (2008), Odu et ál. (2013) entre otros, ya que actualmente, por medio de la trazabilidad, se involved in packaging. The risk analysis results indicate that the human factor participating in the packaging obtains the highest risk rating, followed by the cultural management of the crop and, in third place, by the harvesting process. In all processes where the risk was quantified, the results showed values close to or greater than 50 in a 0 to 100 risk scale. These results should alert producers and consumers of produce for fresh consumption in the analyzed population. The effect of the buyer on the packaging risk values was also observed; when the producers are supermarket chain suppliers the risk values are lower as compared to those of producers who are not supermarket chain suppliers.

puede determinar la trayectoria de un producto desde su punto de venta hasta el lugar y las personas que lo manipularon en la etapa de producción (Fulponi 2010).

Existen diferentes trabajos de investigación que evalúan metodologías para detectar riesgos de contaminación en los alimentos. Algunas de las más recientes se basan en el uso de biosensores (Bhunia 2008) o como el caso de Andrew et ál. (2011), quienes proponen la técnica de Multipexed, la cual logra una mejora importante en los tiempos de detección de contaminantes en los alimentos. Estos esfuerzos son importantes, ya que permiten por medio de una evaluación, detectar los posibles contaminantes a una mayor velocidad y así evitar que los productos contaminados lleguen a las personas, pero a la vez tienen inconvenientes, ya que esta Evaluación de Riesgos Microbiologicos (MRA por sus siglas en inglés) según lo cita FAO (2008), requiere de una importante inversión en recursos, que reduce así la factibilidad de aplicarlos frecuentemente.

Tanto la FAO como la OMS promueven actualmente un abordaje del tema más holístico, enfocado en la seguridad de los alimentos, en el cual se contempla toda la cadena alimenticia para 
lograr alimentos más seguros al ser consumidos y que a la vez pueda ser implementado de una forma ágil (FAO 2008).

El análisis o evaluación de riesgos, es una herramienta utilizada ampliamente en la agricultura, tal y como lo indican Just et ál. (2010), por lo tanto, su aplicación a la contaminación de alimentos podría permitir llegar a ese objetivo holístico que persiguen la FAO y la OMS, ya que haría posible organizar la información para entender mejor la interacción entre microorganismos, alimentos y enfermedades humanas; además permitiría comparar diferentes escenarios y optimizar la toma de decisiones enfocadas en mejorar una determinada situación de riesgo, que reduce así la probabilidad y el impacto de la contaminación antes de que sucedan, en lugar de únicamente detectar los alimentos que están o no contaminados, tal y como sucede cuando se usan las técnicas antes citadas (FAO 2008).

La FAO (2008) propone la herramienta de análisis de riesgos como una posibilidad para gestionar los riesgos de contaminacion de alimentos; a la vez plantea que no se tiene definido con claridad un método para realizar el análisis del riesgo en los procesos productivos de los alimentos, lo que aduce que existe alta variabilidad entre una enorme lista de factores que podrían influir sobre los resultados. Dentro de estos factores altamente variables, menciona factores ligados al sistema de producción, ambientales determinados por la geografía, fisiológicos de los cultivos y tal vez uno de los más importantes, la intervención humana, ya que este factor es una de los principales fuentes de contaminación que influye de manera independiente en las operaciones e insumos propios del proceso de producción y a su vez, puede modificar el medio para potencializar la contaminación y transmitirla de un lugar a otro (Mei et ál. 2013).

\section{Análisis de riesgos como herramienta de solución a la problemática}

$\mathrm{Al}$ abordar el tema de riesgo, es importante recordar algunas definiciones. La vulnerabilidad se define como la existencia de peligros en cualquier acción que las personas se propongan desarrollar. Por otro lado, al hablar de peligros, se hace mención a situaciones que causan un daño cada vez que suceden. Es importante aclarar que no es lo mismo un peligro que un riesgo (Mei et ál. 2013, Espinosa 2012).

Aún cuando normalmente se utilicen riesgo y peligro como sinónimos, es necesario aclarar que el riesgo es el enfoque moderno de la previsión y control de las consecuencias futuras de la acción humana y por otro lado, el peligro está dado por la probabilidad de que suceda un evento que causa un daño (Espinosa 2012).

Los riesgos son una gestión de los peligros, si no se gestiona un riesgo, una acción dada puede ocurrir en cualquier momento. Por lo tanto, un peligro pasa a ser un riesgo cuando se gestionan acciones para que no ocurra (Espinosa, 2012). Este planteamiento concuerda con el enfoque holístico que desean desarrollar la FAO y la OMS, de no solo cuantificar los peligros sino gestionarlos por medio de un análisis de riesgos (Espinosa 2012).

Cuando se va a realizar un análisis del riesgo, se deben desarrollar 3 etapas: identificación, estimación y análisis y evaluación. Si se conocen los posibles peligros asociados a un sistema de producción, si se cuantifican y categorizan, se puede planificar la gestión de los mismos y así anticipar las causas y posibles efectos. Además permite disponer de insumos para trabajar el diseño de las acciones más apropiados para tratarlos (Espinosa 2012).

La presente investigación tiene como objetivo caracterizar el riesgo involucrado para cada uno de los procesos de producción de hortalizas de consumo fresco, en el Valle Central, en Costa Rica.

\section{MATERIALES Y MÉTODOS}

La identificación y la cuantificación de los riesgos asociados a los diferentes procesos de producción y a los recursos humanos utilizados dentro de los sistemas de producción de hortalizas para consumo fresco, se realizó por medio de un muestreo en las áreas de producción de hortalizas de consumo fresco en el Valle Central de Costa Rica durante 2012 y 2013. En la Figura 1 


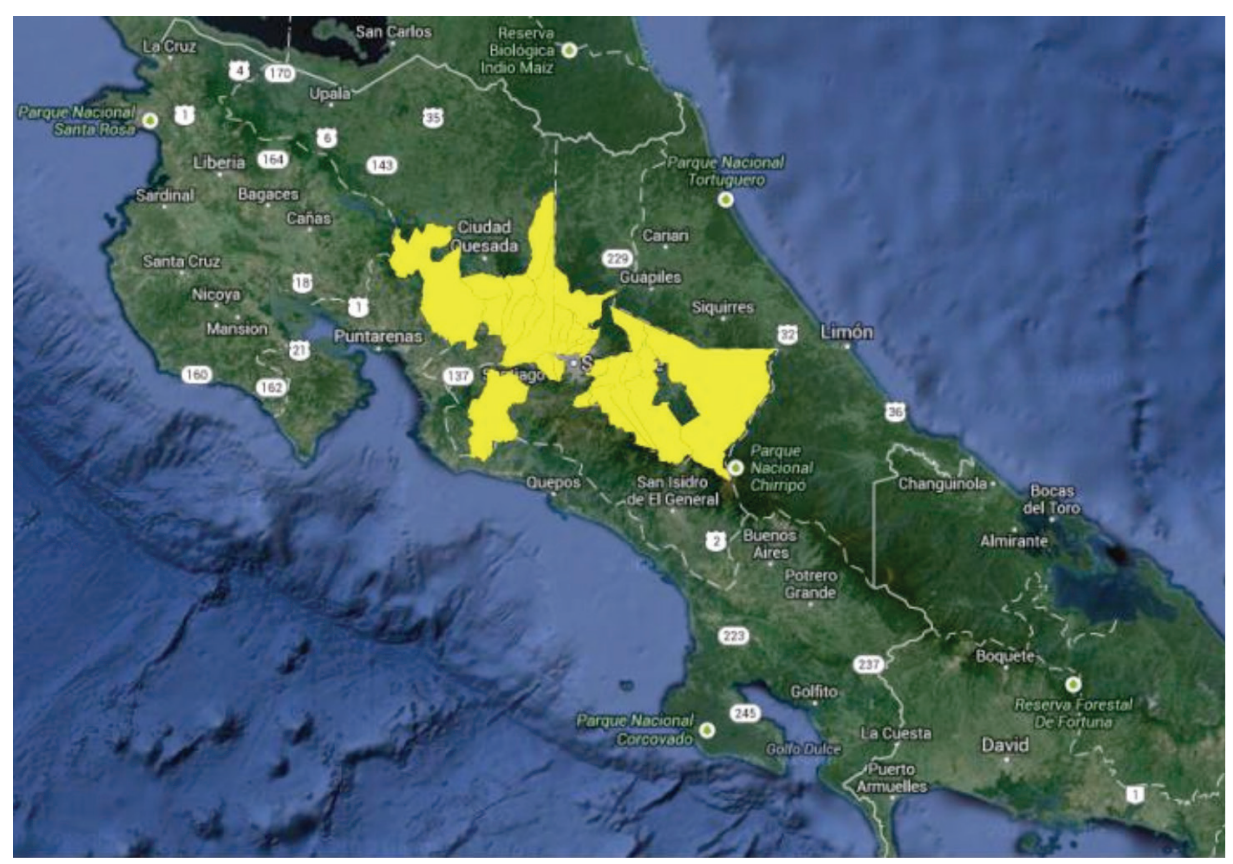

Fig. 1. Zonas geográficas donde se realizaron las entrevistas del diagnóstico de la inocuidad de la producción de hortalizas para consumo fresco. Costa Rica 2012-2013.

se detalla el área de estudio de esta investigación en color amarillo, sobre el mapa del país.

En las zonas donde se llevó a cabo el estudio, los encuestados se clasificaron según los sistemas de producción: al aire libre y bajo ambientes protegidos. No se realizó clasificación según el área productiva u otro tipo de clasificación de tamaño de los encuestados. Los cultivos que se incluyen en esta investigación son: tomate, chile dulce, cebolla, zanahoria, repollo, brócoli, apio, culantro y lechuga.

Dentro de la muestra se pudo identificar productores que comercializan el producto en ferias, al detalle o en centros de acopio y distribuidores mayoristas, así como productores que son proveedores de cadenas de supermercados.

Para esta investigación se utilizó una entrevista de 46 ítems, que fue aplicada en 164 empresas.
Mediante la encuesta, se evalúa el nivel de cumplimiento, por parte de los productores encuestados, de los 46 puntos críticos de control previamente determinados para la producción de hortalizas de consumo fresco. Cada uno de éstos representa un peligro de contaminación del producto con microorganismos patógenos de los seres humanos o con residuos de los plaguicidas aplicados al cultivo. Los 46 ítems de la encuesta se agruparon por proceso y se cuantificó el riesgo para cada uno de ellos. Para esto se realizó una segmentación general del sistema de producción agrícola a partir de los siguientes procesos:

1. Manejo de preparación de terreno y siembra (selección del terreno, tipo de labranza, aplicación de enmiendas químicas, incorporación de materia orgánica, tratamiento de desechos, siembra o trasplante). 
2. Manejo cultural (selección de fuentes de agua para aplicación de riego, sistema de riego implementado, manejo de drenajes, fertilización, podas, aporcas, tutorado, monitoreos o evaluaciones, presencia de animales domésticos).

3. Condiciones y procedimientos seguidos por el factor humano que participa en el proceso de producción (condiciones tales como: capacitación en actividades realizadas y manejo de higiene en las mismas, disponibilidad de servicios sanitarios, áreas de alimentación, agua potable para realizar sus necesidades básicas. Procedimientos tales como: higiene personal, uso de ropa exclusiva para el trabajo, informar sobre el padecimiento de alguna enfermedad infectocontagiosa).

4. Manejo fitosanitario de los cultivos (selección de insumos y fuentes de agua para la preparación y aplicación de insumos para el control de plagas y enfermedades, respeto a período de carencia, uso de equipo adecuado para la manipulación de agroquímicos, uso de equipo adecuado para la medición de agroquímicos, calibración y determinación de dosis).

5. Manejo de la cosecha (tipo y manejo de recipientes de cosecha, lugar de almacenamiento de recipientes de cosecha, procedimientos de lavado de recipientes de cosecha, uso de indumentaria específica para realizar la cosecha, transporte, tratamientos de limpieza del producto cosechado, supervisión de la cosecha).

6. Manejo del empaque (condiciones generales de la planta empacadora, manejo de recipientes de empaque, lugar de almacenamiento de recipientes de empaque, uso de mesas y pilas para el lavado y empaque, tipo de desinfectante para tratar el producto final, lugar de almacenamiento de los productos empacados, procedimiento y registro de las condiciones en que se transporta el producto).

7. Condiciones y procedimientos seguidos por el factor humano que participa en el proceso de empaque (capacitación en empaque y riesgos de contaminación de alimentos, exclusividad en labores de empaque, uso de ropa exclusiva para realizar el empaque, disponibilidad de servicios sanitarios, registros de limpieza de manos y servicios sanitarios, disponibilidad de agua potable, áreas de preparación antes de ingresar al empaque, etc.

A los resultados por proceso de cada una de las entrevistas se le aplicó el "Sistema para la cuantificación de riesgos de contaminación de productos agrícolas" propuesto por Vargas et ál. (2015), el cual cuantifica el riesgo basado en peligrosidad, vulnerabilidad y capacidad de respuesta, para así obtener una estimación global para la población analizada del riesgo de cada proceso.

Tal y como lo indica la FAO (2008), existen diferentes factores que pueden influir en la probabilidad e impacto que pueda tener un peligro en la cadena de producción de un alimento de consumo fresco, entre ellos se destaca el factor humano como uno de los más importantes, es por ello que se incluye en este análisis no solo los procesos como tales, sino que se busca también explorar el aporte específico sobre el riesgo que genera el recurso humano que participa en las etapas de la producción, desde la preparación del terreno y siembra hasta la cosecha, identificado para esta investigación como recurso humano del manejo cultural del cultivo. De igual manera se analizó también el efecto del recurso humano relacionado con el manejo del proceso del empaque.

La escala de calificación del riesgo utilizada va de 0 a 100 , donde las calificaciones cercanas a 0 indican un bajo riesgo y las cercanas a 100 un alto riesgo de pérdida de inocuidad. Los resultados se presentan y analizan por medio de cuartiles. Se parte de que el cuartil 1 es un valor tal que el $25 \%$ de los datos, que en este caso son empresas agrícolas, tienen un valor de riesgo inferior al valor obtenido, y el $75 \%$ de los datos tienen valores de riesgo superior al valor del cuartil 1 . 
El manejo estadístico de los datos se realizó con el paquete estadístico JMP.

\section{RESULTADOS Y DISCUSIÓN}

\section{Determinación del riesgo de contaminación del producto}

A continuación se presentan los resultados del riesgo asociado a cada proceso de producción agrícola de hortalizas para consumo fresco

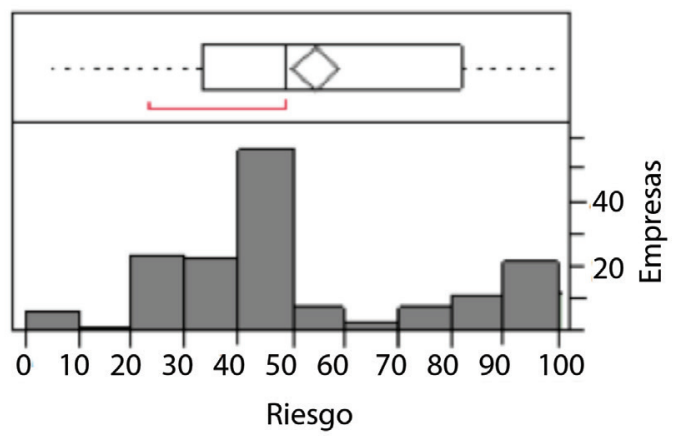

Fig. 2. Distribución de frecuencia del riesgo de contaminación en el proceso de preparación del terreno y siembra de la población evaluada en los diferentes sistemas de producción de hortalizas para consumo fresco en el Valle Central de Costa Rica. 2013.

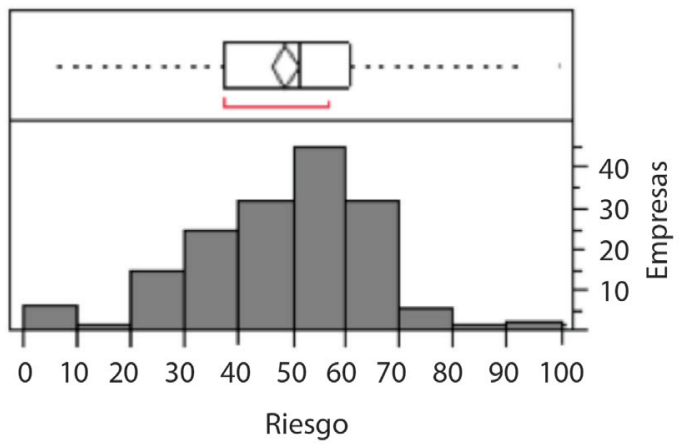

Fig. 4. Distribución de frecuencia del riesgo de contaminación asociado al factor humano ligado al manejo cultural del cultivo de la población evaluada en los diferentes sistemas de producción de hortalizas para consumo fresco en el Valle Central de Costa Rica. 2013. definidos anteriormente. Inicialmente se presentarán las figuras donde se muestra la distribución de frecuencias (número de empresas) del riesgo de contaminación por proceso definido para determinar posibles tendencias de la población evaluada, luego se presenta un cuadro resumen con los percentiles de riesgo de contaminación del producto por proceso el cual aporta un mayor detalle de los resultados obtenidos.

En las Figuras de la 2 a la 8 se presenta la distribución de las empresas en la escala de riesgo

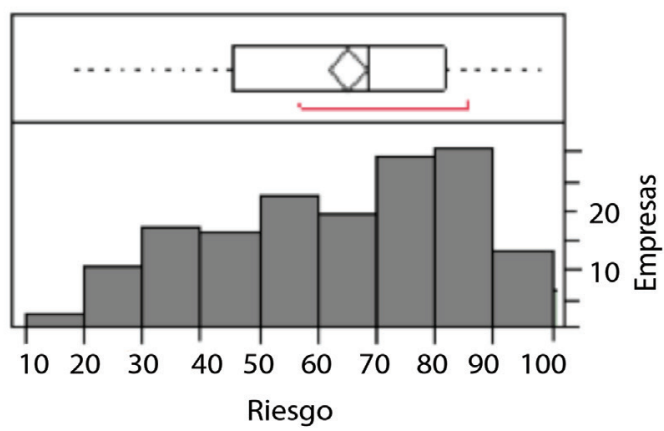

Fig. 3. Distribución de frecuencia del riesgo de contaminación en el proceso de manejo cultural del cultivo de la población evaluada en los diferentes sistemas de producción de hortalizas para consumo fresco en el Valle Central de Costa Rica. 2013.

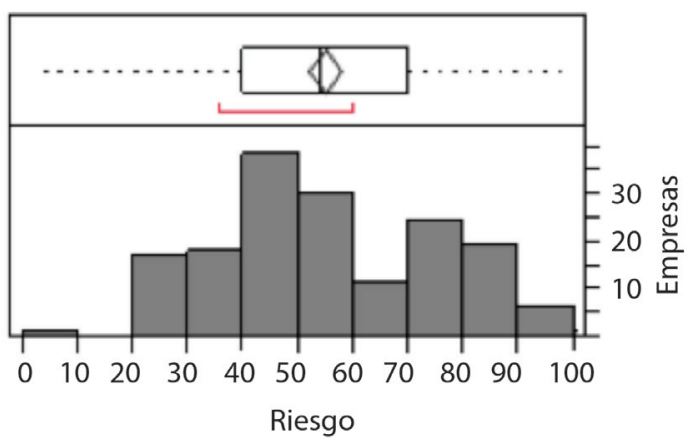

Fig. 5. Distribución de frecuencia del riesgo de contaminación en el proceso de protección del cultivo de la población evaluada en los diferentes sistemas de producción de hortalizas para consumo fresco en el Valle Central de Costa Rica. 2013. 


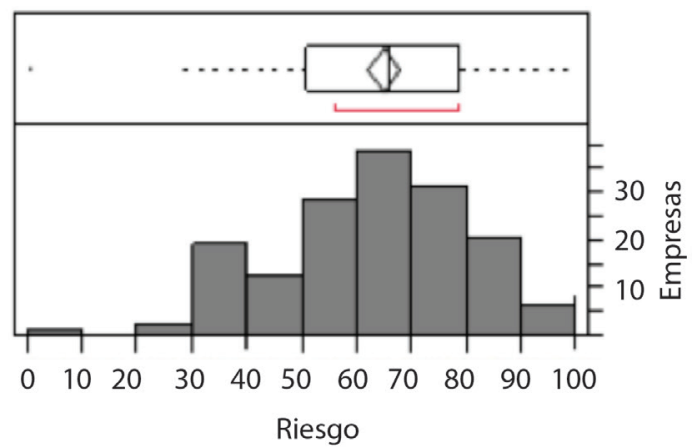

Fig. 6. Distribución de frecuencia del riesgo de contaminación en el proceso de cosecha del cultivo de la población evaluada en los diferentes sistemas de producción de hortalizas para consumo fresco en el Valle Central de Costa Rica. 2013.

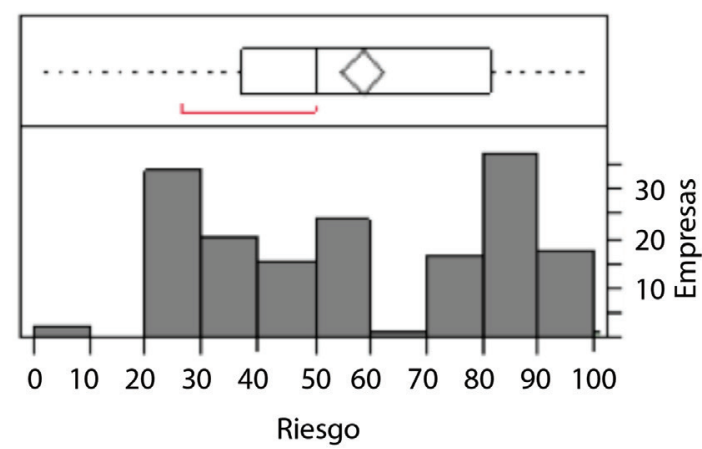

Fig. 7. Distribución de frecuencia del riesgo de contaminación en el proceso de empaque de la población evaluada en los diferentes sistemas de producción de hortalizas para consumo fresco en el Valle Central de Costa Rica. 2013.

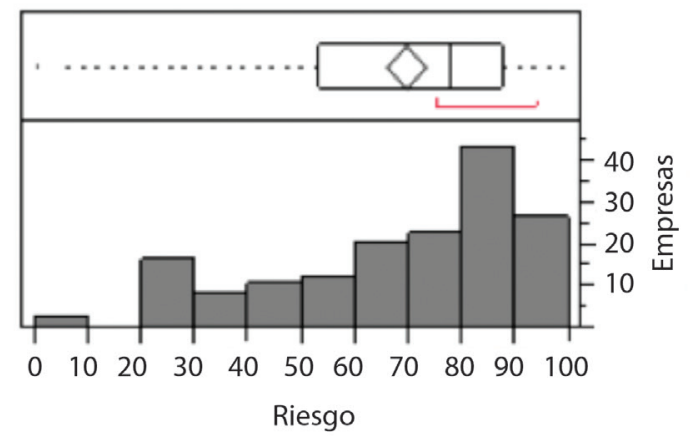

Fig. 8. Distribución de frecuencia del riesgo de contaminación en el factor humano ligado al manejo del empaque de la población evaluada en los diferentes sistemas de producción de hortalizas para consumo fresco en el Valle Central de Costa Rica. 2013. de contaminación para los diferentes procesos analizados. En su mayoría esas figuras muestran empresas en todo el rango de la escala de riesgo. En algunos casos se observa concentración de empresas en los valores medios de la escala es decir de 20 a 50, como el caso de las Figuras 2 y 4 , que representan los procesos de preparación de terreno-siembra y del factor humano ligado al manejo cultural del cultivo.

Otras Figuras como la 3, 6 y 8 concentran empresas en valores de 50 a 100 en la escala, lo que indica que existen empresas con valores de riesgo muy cercanos al límite superior en el proceso de manejo cultural del cultivo, en el proceso de cosecha y en el factor humano ligado al manejo del empaque.

En las Figuras 4 y 8 se incorporan los resultados de este análisis del riesgo de contaminación asociado a las personas que participan en el proceso de manejo cultural y en el empaque. El factor humano es quien administra los insumos y los procesos, es por ello que se realizó un análisis del impacto en el riesgo que incorporan las personas que participan en los procesos de manejo cultural y manejo del empaque tal y como lo proponen Mei et ál. (2013).

En el caso de las Figuras 5 y 7 relacionadas con la distribución de frecuencia del riesgo ligado al proceso de protección de cultivos y empaque, el comportamiento encontrado es diferente al que se ha observado en los procesos anteriores, ya que aunque se observa presencia de empresas en todos los niveles de la escala de riesgo, la distribución es claramente bimodal lo que indica la existencia de al menos 2 poblaciones con características diferentes relacionadas a la variable que se analiza, tal y como lo indica FAO (2008).

El Cuadro 1 incluye en detalle el análisis por percentiles realizado a los valores de riesgo alcanzados por la población muestreada para cada uno de los procesos de producción definidos.

Según los resultados del Cuadro 1 , se debe tener claro que cualquier valor superior a cero, aún cuando se trate de un bajo porcentaje de la población, representa una amenaza a la salud pública. 
Cuadro 1. Valores de riesgo de contaminación en los procesos de producción definidos para los diferentes sistemas de producción de hortalizas de consumo fresco en el Valle Central de Costa Rica. 2013.

\begin{tabular}{rrrrrrrrr}
\hline & \multicolumn{7}{c}{ Proceso } \\
\hline Percentiles & & $\begin{array}{c}\text { Preparación } \\
\text { de terreno y } \\
\text { siembra }\end{array}$ & $\begin{array}{c}\text { Manejo } \\
\text { cultural del } \\
\text { cultivo }\end{array}$ & $\begin{array}{c}\text { Personal } \\
\text { manejo } \\
\text { cultural }\end{array}$ & $\begin{array}{c}\text { Protección } \\
\text { de cultivo }\end{array}$ & Cosecha & Empaque & $\begin{array}{c}\text { Personal de } \\
\text { empaque }\end{array}$ \\
\hline $100,0 \%$ & máximo & 100,00 & 100,00 & 100,00 & 100,00 & 100,00 & 100,00 & 100,00 \\
$99,5 \%$ & & 100,00 & 100,00 & 100,00 & 100,00 & 100,00 & 100,00 & 100,00 \\
$97,5 \%$ & & 100,00 & 100,00 & 80,35 & 92,00 & 100,00 & 97,37 & 100,00 \\
$90,0 \%$ & & 97,44 & 92,21 & 67,29 & 82,80 & 86,89 & 92,11 & 93,88 \\
$75,0 \%$ & 3er cuartil & 82,05 & 81,82 & 60,51 & 70,00 & 78,69 & 81,58 & 87,76 \\
$50,0 \%$ & mediana & 48,72 & 68,83 & 51,40 & 54,00 & 65,57 & 50,00 & 77,55 \\
$25,0 \%$ & 1er cuartil & 33,33 & 45,13 & 37,38 & 40,00 & 50,82 & 36,84 & 53,06 \\
$10,0 \%$ & & 23,08 & 33,12 & 26,36 & 26,00 & 34,43 & 28,95 & 28,57 \\
$2,5 \%$ & & 7,69 & 24,68 & 5,61 & 22,00 & 34,43 & 23,68 & 20,71 \\
$0,5 \%$ & & 5,13 & 16,88 & 5,61 & 2,00 & 0,00 & 0,00 & 0,00 \\
$0,0 \%$ & mínimo & 5,13 & 16,88 & 5,61 & 2,00 & 0,00 & 0,00 & 0,00 \\
\hline
\end{tabular}

Se observa entonces que, 4 de los 7 procesos aportan resultados superiores a cero en todas de las empresas analizadas, aunque cabe destacar el hecho de que existen algunas pocas empresas con valores de cero en los procesos de cosecha, empaque y del personal asociado al empaque, lo cual indica que en estas últimas etapas del proceso, algunas pocas empresas han puesto su esfuerzo en reducir o eliminar los factores de riesgo, que dejan de lado el posible impacto de las etapas relacionadas con la producción.

Tal y como se puede comprobar en el Cuadro 1, para el proceso de preparación de terreno y siembra, el valor mínimo de riesgo que presenta una empresa es de 5,13 y el valor máximo es 100, existe un 2,5\% de empresas que obtienen valores de 100 en la calificación de riesgo de contaminación. La mediana de la población para este proceso se encuentra en un valor de 48,72 y el tercer cuartil muestra que el $25 \%$ de la población presenta valores de riesgo superiores a 82,05.
Es importante destacar que en este proceso, ninguna empresa evaluada tiene calificación cero. Contrario a lo que se podría esperar en este proceso, se observó valores de riesgo altos ligados al mismo, se debe tener en cuenta que en este proceso influyen factores como la aplicación de fuentes de materia orgánica, las cuales, según la fuente, podrían tener una alta probabilidad de contener microorganismos patógenos de humanos, tal y como lo reportan Duffy et ál. (2004). La utilización de insumos orgánicos mal preparados o con ingredientes de alto riesgo de contaminación, se convierten en un importante riesgo de contaminar la producción de hortalizas de consumo fresco durante el primer proceso de producción.

En el caso del proceso de manejo cultural del cultivo, un valor de 16,8 es el valor mínimo que puede presentar una empresa y el valor máximo es 100. En este proceso, la mediana de la población tiene un valor de 68,83 . Este resultado es 20 puntos superior al valor de riesgo que 
alcanza la mediana de la población en el proceso anterior de preparación del terreno y siembra. Al valorar el tercer cuartil, se observa que existe un $25 \%$ de la población que tiene valores de riesgo superiores a 81,82 . En este caso también se observa que existe solo un $2,5 \%$ de las empresas que obtienen 100 en la calificación de riesgo de contaminación.

Al comparar el proceso de preparación de terreno y siembra versus el proceso de manejo cultural, los resultados muestran que en esta última etapa, las empresas alcanzan valores de riesgo superiores en la escala.

Dentro de los componentes del proceso de manejo cultural se encuentra el sistema de riego del cultivo y el uso de fuentes de agua de riego, lo cual, sin duda es una de las actividades que más riesgo pueden introducir al cultivo.

Según el sistema de riego que se utilice para proporcionar agua a los cultivos, en especial de la particularidad de que el follaje del cultivo se moje durante el riego o no y principalmente de la calidad de agua que se emplee para esta tarea, aumenta o disminuye el riesgo de contaminación.

Se debe tener claro que el agua es un transportador de contaminación por excelencia, además, el agua es uno de los recursos que más contacto tiene con el cultivo y el recurso humano que lo administra. $\mathrm{Ng}$ et ál. (2005) registraron este tipo de situaciones y concluyeron que el agua que se aplica a los cultivos se convierte en fuente de contaminación para los alimentos que se producen.

$\mathrm{Al}$ evaluar el riesgo que aporta el recurso humano que participa en las labores de manejo cultural que se observan en el Cuadro 1, se muestra que no existen empresas con calificaciones de riesgo de 0 , el valor más bajo de riesgo que presenta una empresa es de 5,61 y el más alto es un valor de 100 .

Al evaluar la mediana de la población de empresas estudiadas se observa que el $50 \%$ de la población en estudio, puede presentar valores superiores 51,5 en la escala de riesgo de contaminación. Al analizar el tercer cuartil, se observa que existe un $25 \%$ de las empresas estudiadas que tiene valores de riesgo superiores a 60,51. En este caso además, solo un $0,5 \%$ de la población presenta valores de riesgo iguales a 100 .

El comportamiento de los valores del primer cuartil, mediana y tercer cuartil explican el comportamiento observado en la Figura 4, donde se muestra una concentración de empresas en el rango de datos de la escala de 30 a 70 .

Aspectos como la ausencia de letrinas en los campos de cultivo, así como la ausencia de agua potable y desinfectante de manos o en general falta de higiene, son algunos de los factores que más influyen en el valor de riesgo de contaminación obtenido, tal y como lo indican Morales et ál. (2009).

Debe considerarse que el riesgo propio del manejo cultural del cultivo y el inherente a la participación del recurso humano se dan en el mismo lugar y al mismo tiempo durante la realización de las tareas propias de este proceso de la producción. A nivel práctico, el análisis de riesgo cada uno por separado, podrían colaborar en la priorización de mejoras para reducir el riesgo en esta etapa de producción.

$\mathrm{Al}$ evaluar el proceso relacionado con la protección de cultivos, se observa que en este caso, tampoco existen empresas con calificaciones de riesgo iguales 0 , el valor más bajo de riesgo que puede llegar a tener una empresa es de 2,0 y el más alto es un valor de 100 .

En este caso se repite el patrón de comportamiento de los anteriores procesos, donde por desgracia, no existen empresas con niveles de riesgo cero en este proceso. Este tipo de patrón de comportamiento de las empresas, podría poner en riesgo la salud de las personas que consumen los productos que las empresas encuestadas producen.

El proceso de protección del cultivo se tiene definidas claramente 2 fuentes claras de contaminación: la química asociada a los plaguicidas y la microbiológica asociada a la calidad del agua usada en la aplicación. De ellas, la fuente de contaminación química es la que comúnmente se asocia con mayor impacto, pero no se puede dejar de lado los factores microbiológicos asociados a 
este proceso. Es conveniente, por lo tanto, enfocarse en ambos factores, tanto el químico y el microbiológico, ya que con frecuencia se utilizan fuentes de agua contaminada para hacer aplicaciones de plaguicidas.

Otros factores ligados al manejo de agroquímicos, tales como calibración de equipos, cumplimiento de periodo de suspensión o carencia, dosificación, calidad microbiológica y química del agua para la aplicación de agroquímicos son algunos de los factores que influyen sobre los resultados del riesgo y la variabilidad entre las empresas evaluadas. Algunos de estos temas son tratados por Ng et ál. (2005), Lopez et ál. (2013) y Pergher y Petris (2008) quienes indican riesgos en el desconocimiento de algunos de los anteriores factores y la reducción de riesgos que se pueden esperar al conocer y manejar correctamente los agroquímicos.

El comportamiento del riesgo en el proceso de cosecha, presenta una diferencia importante al comportamiento de los procesos anteriores, ya que en este caso al menos un 0,5\% de las empresas presentan calificaciones de riesgo iguales a 0 , aunque $2,5 \%$ de las empresas presentan valores de riesgo máximo de 100. Lamentablemente este resultado respalda el alto riesgo que está presente en este proceso de producción, pero a la vez, se muestra que si es posible llegar a la meta de reducir al máximo los riesgos de contaminación.

Recursos como cajas sucias o acciones como colocar el producto cosechado en el suelo, así como el contacto con la ropa contaminada de los cosechadores, son algunos factores que aumentan el riesgo de contaminación del producto en este proceso.

Los peligros de contaminación del producto para consumo fresco en el proceso de cosecha, incorporan mayor riesgo, ya que existen recursos y acciones que podrían contaminarlo, en una de las últimas etapas antes de que lleguen a ser consumidos. Además, este proceso concentra el producto para consumo fresco en recipientes o lugares, por lo que, si se diera una contaminación en este proceso, afectaría a una mayor cantidad de producto y se distribuiría en diferentes lugares a través de la cadena de comercialización.

Basados en los resultados del Cuadro 1 y Figura 7, se observa como el proceso de empaque presenta un comportamiento diferente al que se ha observado en los procesos anteriores, ya que como se mencionó anteriormente, presenta 2 concentraciones de empresas en 2 puntos diferentes de la escala de riesgo.

En este proceso también se repite el comportamiento de empresas con calificaciones de riego iguales a 0 y de la misma forma existe un 0,5\% de empresas con calificaciones de 100 .

El valor de riesgo de la mediana de la población coincide con la mitad del valor de la escala de valor del riesgo, por lo tanto el $50 \%$ de la población puede presentar valores superiores o inferiores a 50, pero este resultado no muestra el comportamiento real de la población ya que como se observa en la Figura 7, existen 2 poblaciones que deberían de ser analizadas independientemente para interpretar de una mejor forma la situación de las empresas en este proceso.

Ambos grupos de empresas tienen valores de riesgo importantes pero uno de ellos se caracteriza por ser menor al otro grupo de empresas. Este resultado se refleja al observar el tercer cuartil, en el cual existen valores de riesgo superiores al 81,58 en un $25 \%$ de la población de empresas entrevistadas in situ.

Las condiciones en que se realiza el empaque por lo general no reúnen las condiciones para mantener la inocuidad, tal y como lo documenta y ejemplifica Morales et ál. (2009), donde el uso de agua no potable y el uso de locales abiertos expuestos a contaminación animal, son algunos de los factores que influyen sobre estos resultados de contaminación.

El último de los procesos que se indican en el Cuadro 1, es el relacionado al personal que participa en el empaque.

En este proceso, el valor de riesgo en el primer cuartil es de 53,06. Si se compara este valor en el primer cuartil con los anteriores procesos, se observa como en este proceso las empresas alcanzan los valores de riesgo más 
altos. Al interpretar este resultado, podría afirmarse que un $75 \%$ de las empresas encuestadas presentan valores de riesgo superiores a 50 , lo cual indica el riesgo potencial que se produce en este proceso del empaque con la participación del recurso humano.

Por otra parte, el valor de riesgo en la mediana es de 77,55, lo cual indica que el $50 \%$ de la población puede presentar valores de riesgo superiores al valor de la mediana. En este caso, el valor alcanzado en la mediana, también es el más alto en la escala de riesgo alcanzado por un proceso.

Enfocados en la salud de las personas, un riesgo superior a cero podría causar importantes problemas a nivel nacional, ya que podría impactar diferentes segmentos de la población y de la economía, por lo tanto, observar valores de riesgo como los que se presentan en este proceso, es suficiente motivo para buscar mejoras lo antes posible en este proceso para las empresas encuestadas.

Según los resultados es importante gestionar los peligros que genera el recurso humano que participa en los procesos de empaque. Tradicionalmente el enfoque que se le ha dado al manejo del proceso de empaque ha estado centrado en el manejo del producto como tal y no incluye, en la mayoría de los casos, al personal que realiza la labor. Es decir en algunos casos existen esfuerzos por mejorar las condiciones físicas del lugar donde se lleva a cabo el empaque, lo que reduce así los riesgos en este proceso, tal y como lo mostró la Figura 7 con el grupo de empresas que se encuentran entre el rango de riesgo de 20 a 30.

Al contrastar este resultado con lo que muestra la Figura 8, se puede evidenciar que los riesgos ligados al recurso humano en el empaque no tienen el mismo comportamiento de la Figura 7, por lo tanto, al realizar esta comparación se observa que indiferentemente de que se realicen o no mejoras en las condiciones del empaque, existe un importante riesgo de contaminación ligado a las personas que participan en el empaque.

A modo de resumen, la Figura 9 muestra los resultados de la mediana de la calificación de riesgo de contaminación para los procesos analizados. Se observa que los aspectos relacionados con el factor humano relacionado al empaque es el que aporta mayor riesgo en el sistema de producción de hortalizas de consumo fresco en las empresas analizadas. El manejo cultural del cultivo y la cosecha, también aportan cada uno

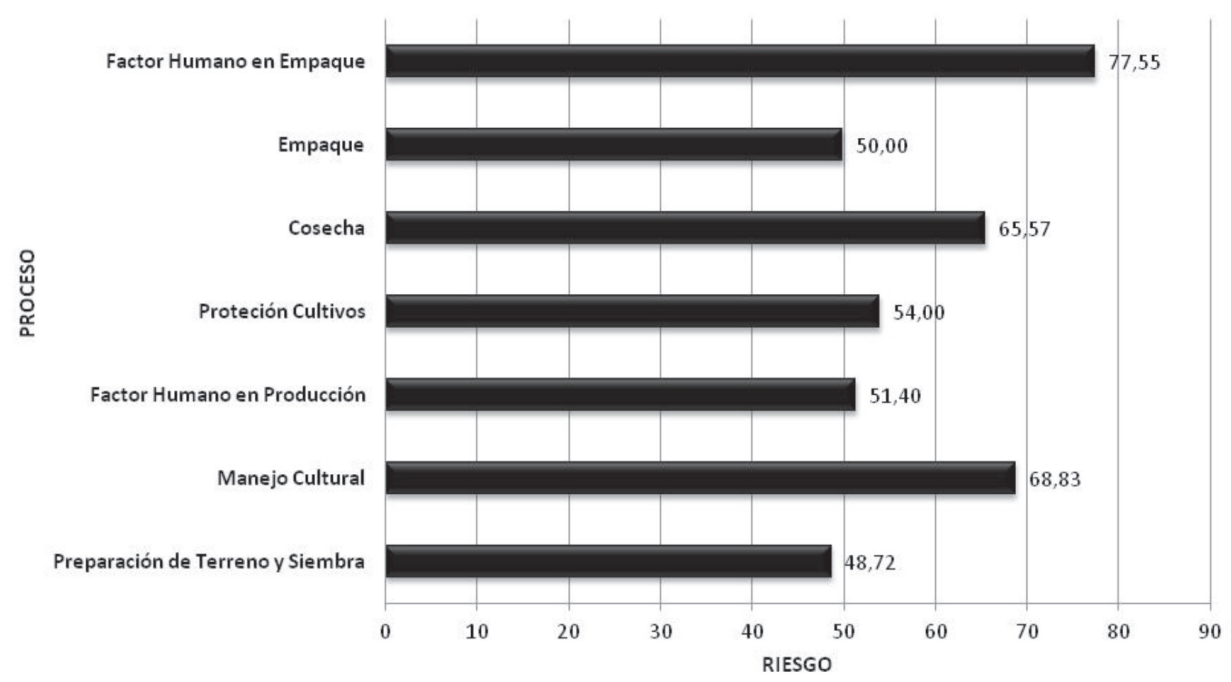

Fig. 9. Resumen de los resultados de la mediana, a partir del resultado del riesgo de contaminación para los procesos y recursos humanos de los sistemas de producción de diferentes hortalizas de consumo fresco evaluados en el Valle Central de Costa Rica. 2013. 
un valor de riesgo importante, por lo que requerirían de mayor atención al momento de planificar acciones para reducir los riesgos de contaminación en las empresas productoras de hortalizas de consumo fresco.

Según lo expone la FAO (2008), el análisis de riesgos es un abordaje que se debe detallar y caracterizar según los factores que intervienen en el sistema, ya que podría existir una gran variabilidad entre éstos, influyendo sobre los procesos y resultados del sistema de producción.

En esta investigación se ha observado la presencia de alta variabilidad en los resultados, se ha usado como fuente de información para hacer este planteamiento las Figuras 2,3,4,5,6,7 y 8, pero además se presenta en la Figura 10 el coeficiente de variación para cada uno de los procesos.

Al analizar en conjunto los resultados del coeficiente de variación (Figura 10), el proceso que mayor variabilidad aporta es el proceso de preparación de terreno y siembra, seguido por empaque. El proceso con menor variabilidad es cosecha. Tanto manejo cultural, personal de campo, protección de cultivos y empaque tienen un comportamiento similar entre ellos.

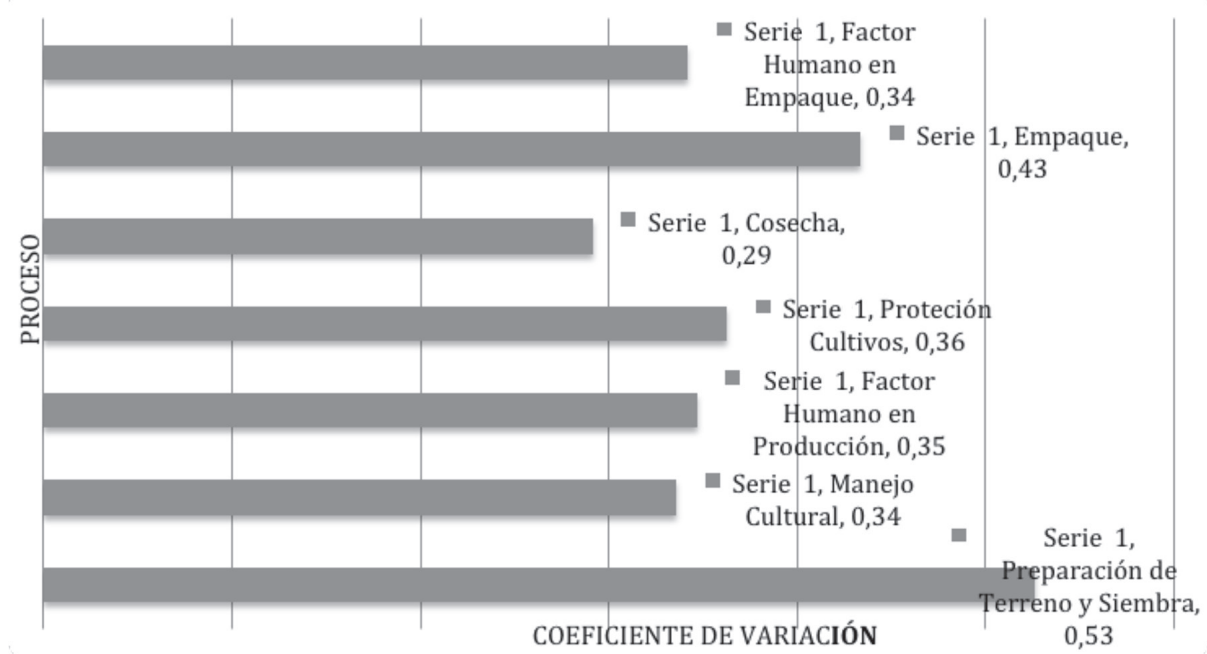

Fig. 10. Resumen de los resultados de la variabilidad, expresado con el coeficiente de variación del resultado del riesgo de contaminación para los procesos y recursos humanos de los sistemas de producción de diferentes hortalizas de consumo fresco evaluados en el Valle Central de Costa Rica. 2013.

Tal y como se comentó anteriormente, este resultado se convierte en un indicador para ligarlo al resultado de la mediana y realizar un análisis integral, tanto del resultado del riesgo de contaminación obtenido, como de la variabilidad que podrían causar los diversos factores que la influyen con el fin de realizar un análisis más profundo de las posibles causas de estos resultados.

De acuerdo con lo que se ha mencionado, estos resultados justifican la necesidad de normalizar los procesos de producción de las hortalizas para consumo fresco, mediante el uso de certificaciones de cumplimiento de las Buenas Prácticas Agrícolas, de modo que logren reducir los valores de riesgo en el producto suplido a los mercados y reducir la variabilidad del riesgo entre la problación de productores proveedores de estos productos.

Un sistema de producción potencialmente riesgoso a la salud humana, disminuye la competitividad de la empresa que lo lleva a cabo, ya que en caso de una contaminación, el 
consumidor podría generalizar sus conclusiones sobre la calidad del producto, sobre los productores y el lugar de origen.

Si bien el análisis de determinación de riesgos por procesos propuesto por los autores mantiene la problemática planteada por FAO (2008), se demuestra que el análisis de riesgo se puede realizar para tomar decisiones de mejoramiento según los datos obtenidos, si se contemplan los valores de las medianas y se relacionan con los valores del coeficiente de variación, con el objetivo de entrar en el análisis a detalle de los factores que pueden influir en los resultados que pueden ser orientados para generar mejoras.

Un ejemplo de la propuesta de análisis que se plantea, es el caso del proceso de empaque, en el cual se observó una situación particular, en donde se presume la existencia de 2 grupos de empresas en las cuales los resultados del riesgo muestran 2 situaciones al parecer diferentes.

\section{Influencia del mercado meta del producto sobre los niveles de riesgo de contaminación}

Como producto de la recolección de datos en campo y el análisis del riesgo de contaminación, se observo que el riesgo de contaminación en el proceso de empaque presenta un comportamiento bimodal, por lo tanto se analizó más a fondo las causas de este comportamiento y se logró relacionar con el merccado meta de los productores. Las empresas entrevistadas se clasificaron en 2: productores que suplen a cadenas de supermercados y los productores que no son suplidores de cadenas de supermercado, es decir que entregan su producto en ferias o mercados tradicionales.

En la Figura 11, se puede observar de forma gráfica la existencia de diferencias en los resultados de las calificaciones de riesgo, obtenidas en el análisis del proceso de empaque.

Los resultados observados en la Figura 11, muestran que en la mayoría de los casos, el riesgo de contaminación en los procesos de los sistemas de producción de hortalizas para consumo fresco, es menor cuando el cliente del productor es una cadena de supermercado al comparar con los resultados de riesgo cuando el cliente no es una cadena de supermercados. Lo anterior es consecuencia de la obligatoriedad que imponen las cadenas de supermercados, a que sus suplidores

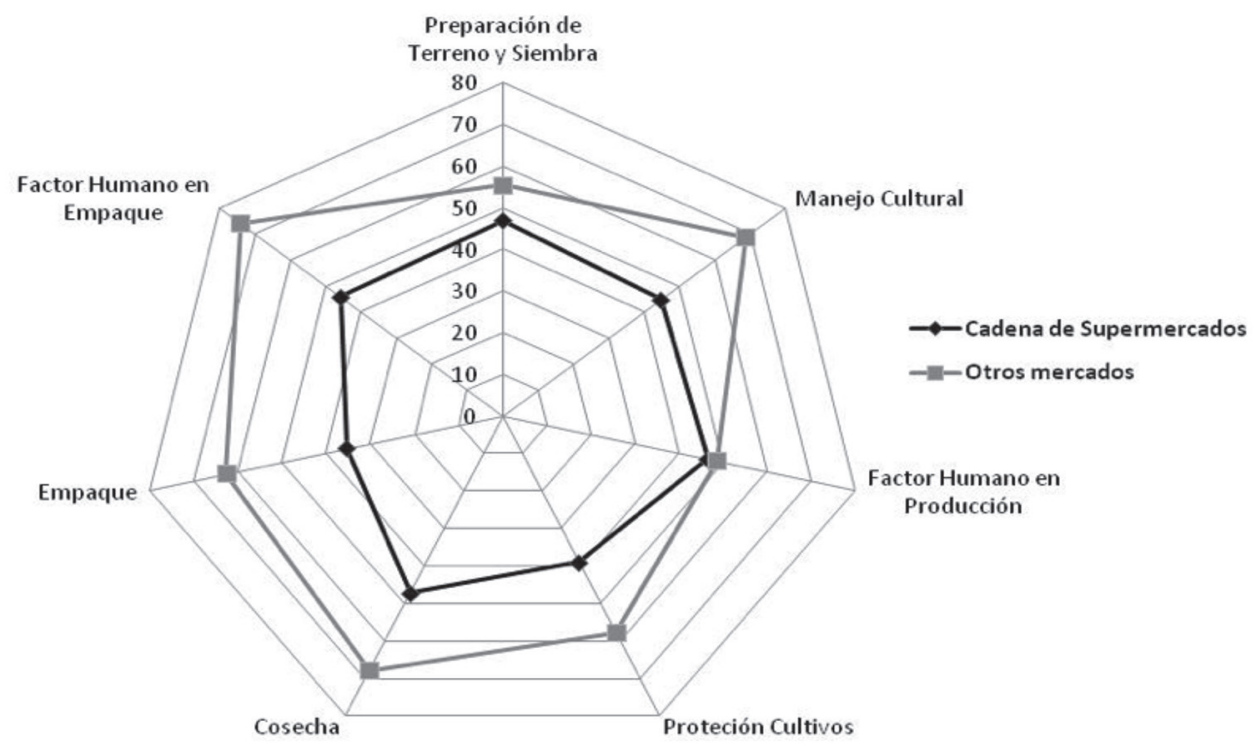

Fig. 11. Comparación de los resultados de riesgo de pérdida de inocuidad para los procesos del sistema de producción de diferentes hortalizas de consumo fresco según el mercado meta del productor. Valle Central de Costa Rica. 2013. 
cumplan a cabalidad la normativa de Buenas Prácticas Agrícolas, así como de la auditoría de dicho cumplimiento.

Este resultado resalta el efecto que tienen las normas de calidad solicitadas por los supermercados a sus proveedores, los cuales los inducen a una implementación de Buenas Prácticas Agrícolas (BPA), lo que se refleja en productos con menor riesgo de contaminación, ya que parte de las funciones de este tipo de códigos de conducta es identificar y gestionar los peligros en el proceso de producción, sin embargo aún bajo estas condiciones, se determinaron valores de riesgo que son un peligro para la salud pública.

\section{CONCLUSIONES}

Dado que el resultado más bajo de riesgo de contaminación detectado en los procesos de producción de la hortalizas para consumo fresco de las empresas analizadas tiene una mediana con un valor de 48,72 se puede concluir que esto representa un peligro para la salud pública y además una serie de oportunidades de mejora en todos los procesos evaluados.

El factor humano que participa en empaque y el manejo cultural, son los procesos que mayor riesgo aporta a los sistemas de producción evaluados.

El aporte determinado por este estudio del recurso humano a los riesgos del proceso de manejo cultural del cultivo y el del empaque, muestran la necesidad de enfocar los esfuerzos no solo en el mejoramiento de las condiciones generales de las fincas de producción de hortalizas, sino también en la capacitación y el control del recurso humano que participa en la cadena de producción de las hortalizas para consumo fresco.

Las exigencias de cumplimiento de las BPA, solicitadas por las cadenas de supermercados del país a los productores proveedores de hortalizas de consumo fresco, según los resultados obtenidos en la investigación, reducen el riesgo de contaminación en los diferentes procesos, aún así, los resultados pueden considerarse peligrosos para la salud pública.
Los niveles de variabilidad en la calificación del riesgo entre las empresas estudiadas y la ausencia de sistemas de trazabilidad en los mercados de hortalizas para consumo fresco justifican la exigencia de normas de calidad que protejan la competitividad de todos los suplidores de un mercado en particular.

El sistema de cuantificación de riesgos por proceso del sistema de producción agrícola, permite obtener resultados que valoran el riesgo de contaminación, que deben ser interpretados en conjunto con los resultados del coeficiente de variación, con el objetivo de identificar la influencia de factores claves que influyen sobre los resultados de la población de empresas que se evaluó en este estudio.

\section{LITERATURA CITADA}

AMEKAWA Y. 2009. Reflections on the growing influence of Good Agricultural Practices in the Global South. Journal of Agricultural and Enviromental Ethnis 22:531-557.

ANDREW G., GEHRING S. 2011. High Throughput Biosensors for Multiplex Food-Borne Pathogen Detection. Annu. Rev. Anal. Chem. 4(1):51-72.

BHUNIA A. 2008. Biosensors and bio-based methods for the separation and detection of foodborne pathogens. Adv. Food Nutr. Res. 54:1-44.

BROWN D.L. 2009. Food Availability and Quality: Situations and Opportunities in Developing Countries, pp. 405413. In: D. Wilson, G. Pond, and B.L.L. Brown, Adequate Food for All. CRC Press.

DUFFY B., SARREAL C., RAVVA, S., STANKER L. 2004. Effect of molasses on regrowth of E. coli O157:H7 an Salmonella in compost tea. Compost Science \& Utilization 12:93-96.

FAO. 2008. Exposure assessment of microbiological hazards in food. Microbiological Risk Assessment Series. Seattle USA: FAO. $102 \mathrm{p}$.

ESPINOSA F. 2012. Procedimiento para evaluar el riesgo de la innovación en la gestion de mantenimiento industrial. Revista Chilena de Ingeniería 20(2):242254.

FULPONI L. 2010. Impacto de los estándades y regulaciones sobre las cadenas agroexportadoras a nivel mundial. Impacto de las Regulaciones y Normas Internacionales de Calidad, Talca: GLOBAL G.A.P. pp. 1-31.

JUST R., DAVID K., SIVALAI J., RICHARD E. 2010. Empirical Changes for Risk Preserences and Production. Annu. Rev. Resour. Econ. 2:13-31. 
HERZFELD T., DRESCHER L.S., GREBITUS C. 2011. Cross-national adoption of private food quality standards. Food Policy 36:401-411.

LOPEZ G., TOMAS A., DIRIBSA D. WEI P. SUSLOW T.V. 2013. Growth of Salmonella enterica in foliar pesticide solutions and its survival during field production and postharvest handling of fresh market tomato. Journal of Applied Microbiology 114 (5):1547-1558.

MEI S., JAN B., RICHARD N. 2013. Perceptual and Actual Risks and How We Comunicate Them, pp. 189-208. In: S. Mei, B. Jan and N. Richard (eds.). Managing Food Safety Risks in the Agri-Food Industies. CRC Press.

MORALES H., HERNÁNDEZ A., QUIROZ R., SUSLOW V. 2009. Detection of Salmonella spp. on Cantaloupe Melon Production Unist and Packing Facility. Agricultura Técnica en Mexico 32(2):135-145.
NG P., FLEET G., HEARD G.M. 2005. Pesticides as a source of microbial contamination of salad vegetables. International Journal of Food Microbiology 237-250.

ODU N., NMA O., ORUESE M. 2013. Prevalence of Salmonella species and Escherichia coli in fresh Cabbage and Lettuce sold in Port Harcourt Metropolis. Report and Opinion. pp. 1-8.

PERGHER G., PETRIS R. 2008. Pesticide dose adjustment un vineyard sprayin and potential for dose reduction. Agricultural Engineering International: the CIGR Ejournal. Manuscript 10:1-9.

PORTER M.E. 2009. Estrategia Competitiva: Técnicas para el análisis de las empresas y sus competidores. Madrid: Piramide, $456 \mathrm{p}$.

VARGAS G., GONZÁLEZ M.I., DURÁN A. MORA D. 2015. Diseño y validación de un sistema para cuantificar riesgos de contaminación y su aplicación para identificar puntos críticos de control en cultivos hortícolas en Costa Rica. Agronomía Costarricense. 39(2):153-166. 
